\title{
Monetite promoting effect of citric acid on brushite cement setting kinetics
}

\section{E. Şahin* and M. Çiftçioğlu}

\begin{abstract}
Brushite forming calcium phosphate cements receive growing interest in hard tissue scaffold applications due to their high surface area and high bioresorbability. The finer microstructure of monetite, the dehydrated form of brushite, has attracted attention for bone tissue engineering applications. The reduction in brushite content of the $\beta$-tricalcium phosphate-monocalcium phosphate monohydrate cement system by selective inhibition of growth upon addition of citric acid to excess setting liquid was investigated. The relaxation period during cement setting was monitored by $\mathrm{pH}$ stat titration and free drift runs. Spectrometric analysis revealed that the change in solubility of calcium phosphates upon addition of citric acid caused the inhibition of brushite formation and promotion of monetite precipitation. Dissolution of monetite crystals was insensitive to citrate adsorption despite their lower surface area compared to brushite. Overall brushite/ monetite ratio decreased consistently with increasing citric acid concentration in the of $0 \cdot 1-0.5 \mathrm{M}$ range.
\end{abstract}

Keywords: Calcium phosphate cement, Brushite, Monetite, Tricalcium phosphate, Citric acid, Titration, Kinetics, Setting

\section{Introduction}

Calcium phosphates are the main class of ceramics used in dentistry, ${ }^{1}$ maxillofacial surgery ${ }^{2}$ and bone tissue engineering ${ }^{3,4}$ because of their similar chemical, physical and structural properties with hard tissues. ${ }^{5}$ Calcium phosphate cements (CPCs) were developed in the 1980s as promising tissue engineering materials that have high resorbability, high surface area and sufficient strength to be used in vivo. Calcium phosphate cements precipitate via an acid-base reaction between acidic and basic calcium phosphate precursors. The setting reaction of brushite cement using slightly basic $\beta$-tricalcium phosphate $(\beta$-TCP) and acidic monocalcium phosphate monohydrate (MCPM) as precursors and its transformation to slightly acidic monetite occur according to the following stoichiometry

$$
\begin{aligned}
& \beta-\mathrm{Ca}_{3}\left(\mathrm{PO}_{4}\right)_{2}+\mathrm{Ca}\left(\mathrm{H}_{2} \mathrm{PO}_{4}\right)_{2} \cdot \mathrm{H}_{2} \mathrm{O}+7 \mathrm{H}_{2} \mathrm{O} \\
& \rightarrow 4 \mathrm{CaHPO}_{4} \cdot 2 \mathrm{H}_{2} \mathrm{O} \\
& 4 \mathrm{CaHPO}_{4} \cdot 2 \mathrm{H}_{2} \mathrm{O} \rightarrow 4 \mathrm{CaHPO}_{4}+8 \mathrm{H}_{2} \mathrm{O}
\end{aligned}
$$

Brushite crystallises in a non-centrosymmetric monoclinic structure with a plate-like morphology dominated by (010) faces, which are fully hydrated. Each calcium ion is bonded to eight oxygen atoms within the crystal, six from neighbouring phosphates and two from water

Department of Chemical Engineering, Izmir Institute of Technology, Gülbahçe Urla, İzmir 35430, Turkey

*Corresponding author, email erdemsahin@iyte.edu.tr molecules. The crystal structure consists of two parallel layers that are composed of sheets of calcium phosphate molecules and water molecules. ${ }^{6}$ The hydrated layer is the terminating layer at the brushite crystal surface in aqueous solutions due to the much higher concentration of water molecules compared to those of calcium and phosphate ions. The (110) plane is terminated by a calcium ion, making it susceptible to adsorption of impurities like citrate group. ${ }^{7}$ Water layer is also interrupted at the step edges on the (010) face, where oxygen atoms are not available from neighbouring phosphates, so that calcium and phosphate ions are able to interact with the surrounding medium, allowing the dissolution of these ions and impurity adsorption. ${ }^{8}$ The growth kinetics is mainly controlled by phosphate incorporation, and additives interfering with this step regulate precipitation and crystal growth. Adsorbed atoms from the solution have to be removed during crystal growth to accommodate the competing $\mathrm{HPO}_{4}^{2-}$ ion; hence, dehydration or impurity deadsorption is an important part of the activation barrier for growth and dissolution. ${ }^{7}$ Monetite on the other hand crystallises in a triclinic unit cell consisting of $\mathrm{CaHPO}_{4}$ chains bonded together by $\mathrm{Ca}-\mathrm{O}$ bonds and three types of hydrogen bonds. ${ }^{9}$ Its stacked sheet-like, compact structure is free from hydration; however, development of a thin apatitic layer in aqueous solutions in 1 week is reported. ${ }^{10} \mathrm{~A}$ schematic representation of the crystal unit cells of both phases based on the crystallography studies by Catti et al. ${ }^{11}$ and Schofield et al. ${ }^{12}$ was adapted in Fig. 1 from a mineralogy database. ${ }^{13}$

Calcium phosphate cement setting kinetics dictate the order and extent of precipitation of the stable phases determined according to thermodynamics. Modification 


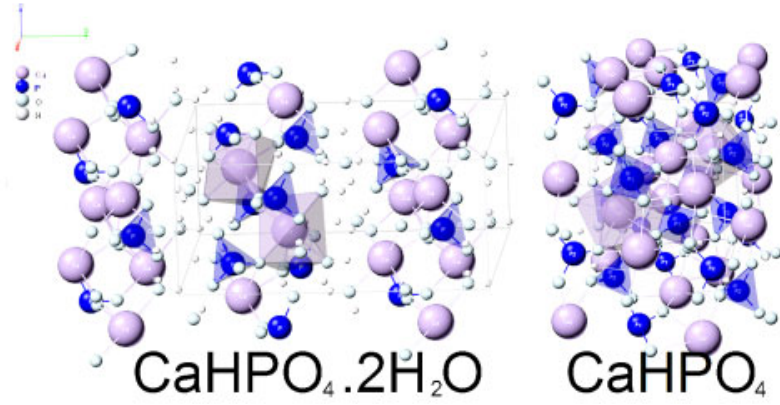

1 Schematic representation of crystal unit cells of brushite $\left(\mathrm{CaHPO}_{4} \cdot 2 \mathrm{H}_{2} \mathrm{O}\right)$ and monetite $(\mathrm{CaHPO})$

of the brushite cement setting kinetics by variation of $\mathrm{pH},{ }^{14,15}$ temperature, ${ }^{16,17}$ water content ${ }^{18}$ and addition of impurities ${ }^{19,20}$ enables obtaining variable phase compositions differing in brushite and monetite content. Monetite formation is generally thought to be favoured by a high temperature and a low $\mathrm{pH}$ value as confirmed by phase analyses of dried cement powders in many studies. Impurities may inhibit both dissolution and growth of the crystals by substituting for similar ions in the crystal, binding to the surface ions with affinity, hindering step motion or chelating free ions with affinity in the solution. ${ }^{21}$ Nancollas et al. demonstrated the general inhibitory effect of citrate on brushite growth as a function of the citric acid concentration. ${ }^{22}$ Citric acid is commonly utilised to slow down the setting and hence increase the workability and handling of CPCs. It decreases the supersaturation of setting liquid due to its affinity to bind calcium ions. In the presence of citric acid, free calcium ion concentration is significantly reduced. In addition, adsorption of citrate group to calcium at the surface of calcium phosphate crystals decreases their dissolution and the number of active growth sites. ${ }^{23}$ Generally, impurities are ineffectively adsorbed at high supersaturations, as the surface integration rate is too high. ${ }^{24}$ Supersaturation of any precipitating solution should be reduced in order to enhance impurity effectiveness.

In the studies focusing on the modification of brushite cement setting kinetics, control on the extent of monetite formation and the accompanying micropore formation was not demonstrated. ${ }^{14,16,19}$ Monetite formation in brushite cement was commonly regarded as a detrimental degradation reaction due to the increase in microporosity. However, Galea et al. ${ }^{14}$ reported a twofold increase in the compressive strength of $\beta$-TCP blocks after conversion to monetite, and Aberg et al. ${ }^{15}$ reported a gradual increase in mechanical properties with time via monetite formation in brushite cement. The comparatively finer crystal morphology of monetite than brushite seen in Fig. 2 hints these results despite the accompanying microporosity formation. Tailoring of brushite cement phase composition and densification of micropores by biomineralisation via hydrothermal methods are possible once a correlation between monetite content and ionic modifier concentration is established. Role of citric acid as a possible control parameter for monetite formation was investigated in this study. The ionic modifier is expected to alter the saturation and growth of the stable phases in brushite cement. Growth curves and $\mathrm{pH}$ variation of the conventional brushite cement system set in excess aqueous solution containing citric acid were analysed in order to determine the effect of citrate impurity on the setting kinetics. Phase composition of the modified brushite cement and solubility of the stable calcium phosphate phases were monitored for all citric acid concentrations studied. Cement growth rate is expected to decrease significantly at the citrate concentration range and due to excess dilution of the setting liquid. The results of the study will provide cues for controlling monetite content in acidic cement setting applications under sufficiently high crystal growth rates.

\section{Experimental}

Sodium hydroxide uptake of the cement solution in a $\mathrm{pH}$ stat potentiometric titrator (Kyoto Electronic AT510) was monitored in order to reveal the growth kinetics. Effect of impurities on the setting reaction of stoichiometric cement formulation was investigated by adding citric acid to the cement solution at a fixed $\mathrm{pH}$ value of $4 \cdot 2$. This value was chosen for its proximity to $4 \cdot 3$, which is the singular point between monetite and hydroxyapatite to prevent any hydroxyapatite formation. Cement powder mixture consisted of $1.55 \mathrm{~g}$ $\beta$-TCP, $1.26 \mathrm{~g} \mathrm{MCPM}$ and $0 \cdot 1 \mathrm{~g}$ brushite seed. $\beta$-TCP (Sigma 21218) and MCPM (Sigma C8017) precursor powders had median particle sizes $d_{50}$ of 5.5 and $33 \mu \mathrm{m}$ respectively. Citric acid (Aldrich 27490) concentration in $100 \mathrm{~mL}$ of deionised water varied in the range of $0 \cdot 1-$ $0 \cdot 5 \mathrm{M}$. $\mathrm{NaOH}(0 \cdot 1 \mathrm{M})$ (Sigma 221465) was used as

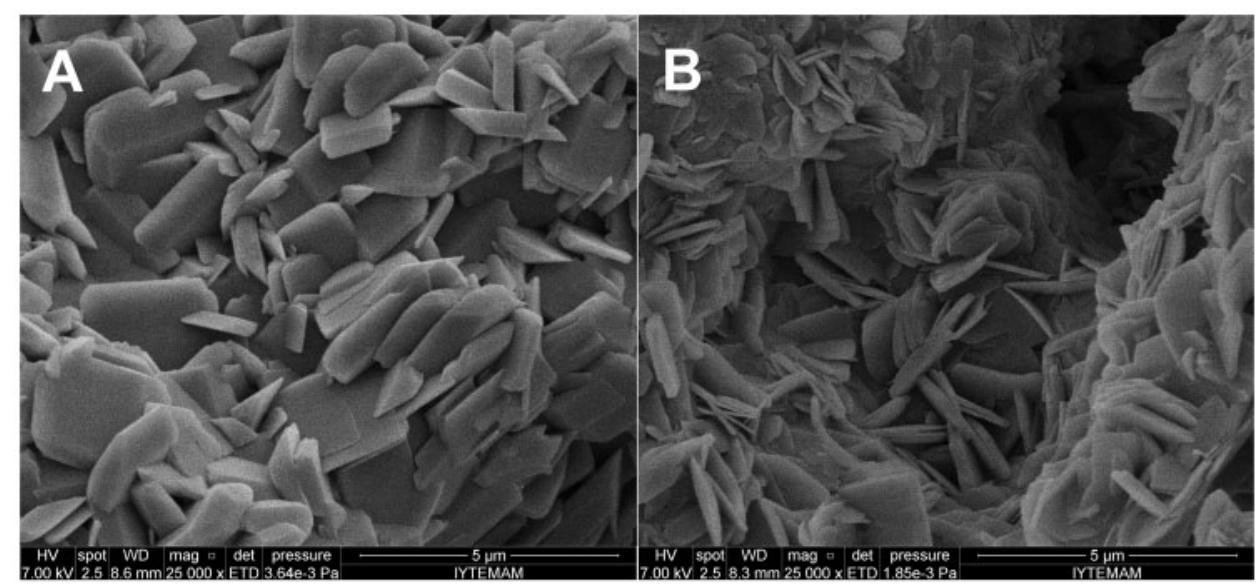

2 Crystal morphology of $a$ brushite and $b$ monetite precipitates obtained using $\beta$-TCP-MCPM cement system 


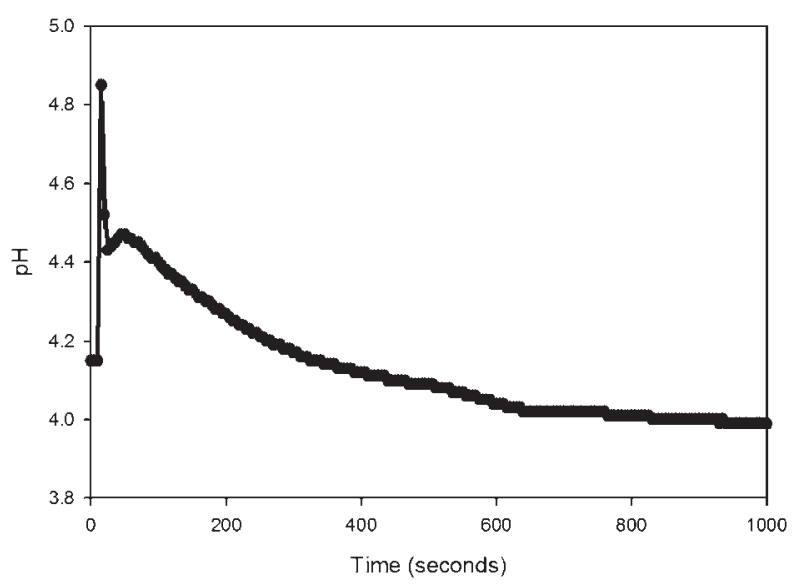

3 Variation in $\mathrm{pH}$ of setting solution for $\beta$-TCP-MCPM cement system with brushite seeds

titration base. Variation of base uptake and the $\mathrm{pH}$ were recorded as functions of time. The observable effects of the ionic modifier citric acid on the kinetics of the cement setting reaction were isolated by keeping other kinetic factors constant. Introduction of seeds reduced the supersaturation build-up, and eliminating variations in temperature and $\mathrm{pH}$ also made it possible to investigate the effects of ionic modifiers on the relaxation period at various concentrations. Free drift runs in addition were conducted under similar conditions in order to observe the variation in $\mathrm{pH}$ as an indication of CPC growth.

The amount of citric acid initially added as planned in the range of $0 \cdot 1-0 \cdot 5 \mathrm{M}$ in the $\mathrm{pH}$ stat runs was diluted by the high volume of concentrated $\mathrm{NaOH}$ added to balance the initial $\mathrm{pH}$ drop caused by citric acid. Kinetics of the reactions was not affected by this dilution as CPC powders were mixed after the removal of extra solution. Only citric acid concentrations in the $\mathrm{pH}$ stat runs decreased slightly. Precipitation products were centrifuged, frozen and freeze dried in order to investigate the microstructure by X-ray diffraction (XRD) using Philips X'Pert Pro powder diffractometer with $\mathrm{Cu} K_{\alpha}$ radiation at a generator voltage of $45 \mathrm{kV}$ and a tube current of $40 \mathrm{~mA}$. All XRD patterns were obtained at a scan range of 5-60 , scan step size of 0.05 and 5 s per step. External standard method was employed for the semiquantitative XRD analysis. Relative intensity ratio of the constituent phases with respect to the external standard corundum was obtained by measurement of the ratio of the intensities of the characteristic peaks to intensities of the characteristic peaks of corundum. The mass absorption coefficients of calcium phosphate phases were obtained from literature. ${ }^{25}$ Characteristic peaks of constituent phases separated by a minimum angle of $1^{\circ}$ were used to characterise the phase composition of the samples by measurement of the net peak intensities. Obtained intensities, relative intensity ratio and mass absorption coefficients were inserted into the governing external standard method equation to obtain weight ratios $^{26}$

$$
W_{\mathrm{a}}=\left(\frac{I_{\mathrm{a}}^{\mathrm{hkl}}}{I_{\mathrm{a}}^{\mathrm{REL}}}\right)\left[\frac{(\mu / \rho)_{\mathrm{m}}}{(\mu / \rho)_{\mathrm{S}}}\right]\left(\frac{1}{I_{\mathrm{S}}^{\mathrm{P}} R I R_{\mathrm{a}}}\right)
$$

where $I_{\mathrm{a}}^{\mathrm{hkl}}$ is the intensity of the $(h k l)$ peak of phase $\alpha$, $I_{\mathrm{a}}^{\mathrm{REL}}$ is the relative intensity of $(h k l)$ peak with respect to other peaks of the same phase, $(\mu / \rho)_{\mathrm{m}}$ is the mass absorption coefficient of the mixture, $(\mu / \rho)_{\mathrm{s}}$ is the mass absorption coefficient of the reference standard, $R I R_{\mathrm{a}}$ is the relative intensity ratio of phase $\alpha$ with respect to the reference standard and $I_{\mathrm{s}}$ is the intensity of the $100 \%$ peak of the reference standard phase $s$, taken by convention to be $\alpha-\mathrm{Al}_{2} \mathrm{O}_{3}$, corundum.

Spectrometric analysis of citrate adsorption on the components of the cement system $\beta$-TCP, brushite (Aldrich 30765-3) and monetite (Sigma C7263) phases was conducted in triplicate sets by using inductively coupled plasma atomic emission spectroscopy and ultraviolet (UV) spectrophotometry in order to determine the free calcium and citrate concentrations in the supernatant upon mixing $1 \mathrm{~g}$ of each component with $100 \mathrm{~mL}$ of citric acid solutions for $24 \mathrm{~h}$. Calcium concentrations of the three phases mixed with varying citric acid solution between 0 and $0 \cdot 5 \mathrm{M}$ were determined using Varian ICP atomic emission spectrometer. Nitric acid (5\%) (Merck, 65\% purity) was added to each sample before analysis to minimise chemical associations and related problems. Citrate concentrations in the supernatant solutions were determined by measurement of absorption at $218 \mathrm{~nm}$ ultraviolet light in a ShimadzuUV 2450 spectrophotometer. Supernatant solutions were diluted to $1: 100$ by adding ultrapure water in order to obtain absorbances within the 0-1 range. Specific surface area or the Blaine value of the three calcium phosphate powders $\beta$-TCP, brushite and monetite were measured by a ToniPERM cement air permeability tester according to the ASTM C204 standard.

\section{Results and discussion}

MCPM instantly dissolves and supplies $\mathrm{H}_{2} \mathrm{PO}_{4}^{-}$and $\mathrm{Ca}^{2+}$ ions to the solution upon mixing brushite cement precursors with excess pure water. A fraction of $\mathrm{H}_{2} \mathrm{PO}_{4}^{-}$ is expected to dissociate to $\mathrm{H}^{+}$and $\mathrm{HPO}_{4}^{2-}$ ions due to its relative stability among phosphoric acid species in water at room temperature. ${ }^{27} \beta$-Tricalcium phosphate dissolves simultaneously to release $3 \mathrm{Ca}^{2+}$ and $2 \mathrm{PO}_{4}^{3-}$ ions, resulting in an initial increase in solution $\mathrm{pH}$ as seen in Fig. 3 due to the hydronium uptake of orthophosphate groups. The $\mathrm{H}^{+}$ions supplied by $\mathrm{H}_{2} \mathrm{PO}_{4}^{-}$ions are expected to increase over time as more $\mathrm{H}^{+}$ions are consumed by dissolving $\beta$-TCP species, and a net decrease in $\mathrm{pH}$ is expected as growth progresses shortly after mixing the reactant with water. Calcium and phosphate ions are rapidly consumed by the growing brushite crystals due to the presence of coherent brushite seeds. The broad peak observed in Fig. 3 of $\sim 100 \mathrm{~s}$ is an indication of the supersaturation build-up by increasing calcium concentration due to fast $\beta$-TCP dissolution at lower $\mathrm{pH}$. The subsequent gradual $\mathrm{pH}$ fall indicates that brushite growth has speeded up relative to $\beta$-TCP dissolution. Precipitation and $\beta$-TCP dissolution act to balance the supersaturation and $\mathrm{pH}$ until the rate of one weakens relative to the other. ${ }^{28}$ This interplay between dissolution and precipitation continues indefinitely until the consumption of precursors. Observed $\mathrm{pH}$ variation curve is parallel to that given in the pioneering work of Bohner et al. on the variation of brushite cement solution $\mathrm{pH}$. The only difference is the initial rise in $\mathrm{pH}$ observed in Fig. 3 due to initial $\mathrm{pH}$ being not kept constant at 4.2 at that study. ${ }^{29}$ Much higher $\mathrm{pH}$ of 


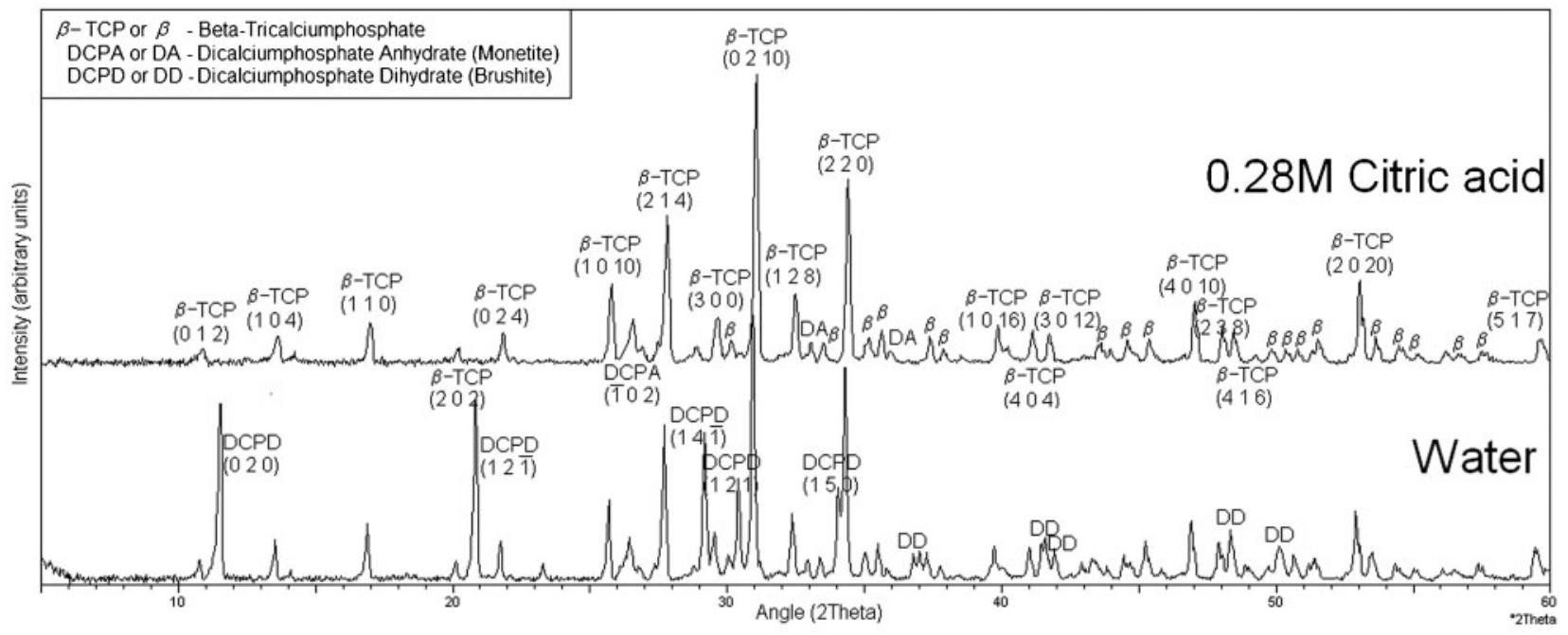

4 X-ray diffraction patterns of precipitates obtained from cement setting in pure water and $0.28 \mathrm{M}$ citric acid solution

dionised water used in Bohner's study decreased the dissolution rate of $\beta$-TCP, so that the peak increasing the equilibrium $\mathrm{pH}$ to 4.8 was not observed. Other observed aspects of cement setting reaction are the same such that a quick drop in $\mathrm{pH}$ is observed due to brushite precipitation, and a broad peak indicative of further $\beta$ TCP dissolution is seen in both curves that reach equilibrium $\mathrm{pH}$ around 4.

The end product of brushite cement setting in pure water under ambient conditions is brushite. The XRD pattern of brushite cement set in excess pure water given in Fig. 4 shows no trace of MCPM and a significant amount of $\beta$-TCP residue. MCPM completely dissolves upon mixing with water, while $\beta$-TCP dissolution continues indefinitely as explained in the above discussion. Phase analysis of precipitates obtained in the titration runs indicates the changes in growth mechanism and kinetics of the system. The weakening of brushite intensity in XRD patterns given in Fig. 4 upon addition of $0.28 \mathrm{M}$ citric acid suggests that brushite growth is kinetically inhibited at the early stages of setting by the interaction of impurities with brushite crystals. Phase composition of the free drift and $\mathrm{pH}$ stat samples given later in the article show that even $0 \cdot 1 \mathrm{M}$

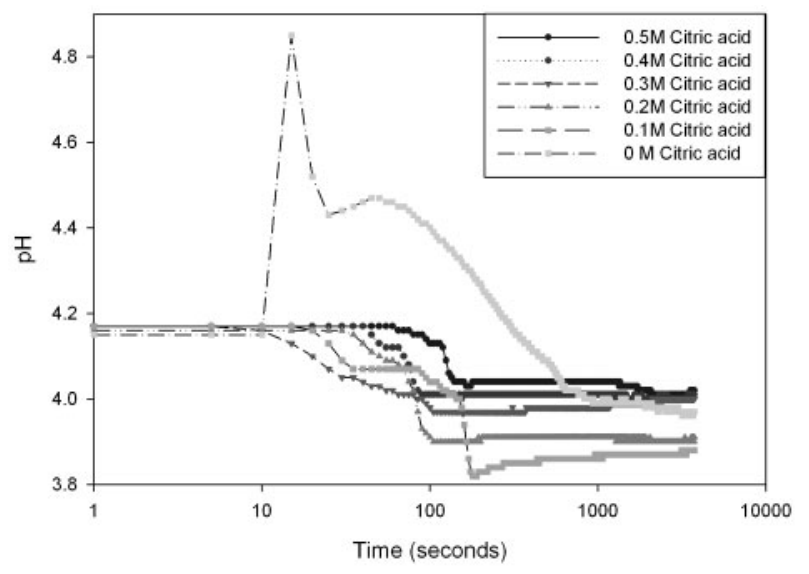

5 Variations in $\mathrm{pH}$ of setting solution for $\beta$-TCP-MCPM cement observed in free drift runs citric acid is sufficient to kinetically favour monetite over brushite. It is seen that monetite peak intensities do not show significant variation in the presence of citric acid compared to pure water. This is attributed to the overall suppression of the growth rates by adsorbing citrate groups on $\beta$-TCP dissolution sites.

\section{Free drift runs}

Free drift analysis of cement setting in water containing 0 to $0 \cdot 5 \mathrm{M}$ citric acid revealed significant differences in the $\mathrm{pH}$ of the solution with varying citric acid concentrations. Final $\mathrm{pH}$ of the cement liquid gradually increased from 3.86 to 4.04 by increasing citric acid concentration from $0 \cdot 1$ to $0 \cdot 5 \mathrm{M}$, as seen in Fig. 5 . The observed effect was not caused by the acidity of citric acid since all cement samples were mixed into aqueous solution at $\mathrm{pH} 4 \cdot 2$. The adsorption of the citrate groups on the dissolving and precipitating crystals and hence the reduction in growth extent is more likely the source of the observed variation in $\mathrm{pH}$. The initial rise of $\mathrm{pH}$ due to the release of $\mathrm{H}^{+}$consuming $\mathrm{PO}_{4}^{3-}$ ions from $\beta$ TCP only occurs in the absence of citric acid. For all citric acid concentrations, a late fall in the $\mathrm{pH}$ corresponding to the dissociation of $\mathrm{MCPM} \mathrm{H}_{2} \mathrm{PO}_{4}^{-}$ groups to $\mathrm{HPO}_{4}^{2-}$ and $\mathrm{H}^{+}$is observed, followed by gradual increase in $\mathrm{pH}$ indicative of supersaturation build-up by further $\beta$-TCP dissolution. Phase compositions given in Fig. 6 reveal that $\beta$-TCP dissolution is greatly reduced with the smallest addition of citrate into the liquid, and the extent of brushite precipitation is lowered with less $\beta$-TCP dissolution.

Phase composition of the precipitates in Fig. 6 obtained from free drift runs shows an intense effect of introducing citric acid in the growth kinetics of the system as the $\beta$-TCP residue significantly increases with $0 \cdot 1 \mathrm{M}$ citric acid addition. Further increasing the citric acid concentration does not affect the $\beta$-TCP dissolution significantly. In addition, the brushite/monetite ratio is seen to significantly decrease with initial citric acid addition in both free drift and $\mathrm{pH}$ stat runs. The results suggest that citrate adsorption on brushite crystal surface is more effective compared to monetite. Such a change in the growth kinetics by impurity addition is expected to make brushite more stable but kinetically less favoured according to Ostwald rule of stages. 


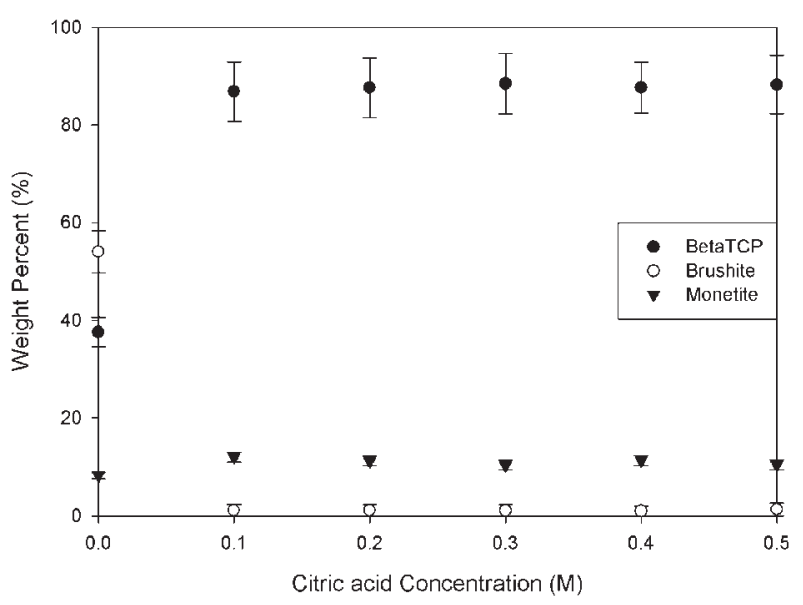

6 Phase composition of precipitate set in liquids containing various citric acid concentrations

Different conclusions are given in literature on the effect of citric acid on brushite/monetite phase equilibria. In a study investigating the shelf life of the $\beta$-TCPMCPM cement system, Gbureck et al. has shown that $0 \cdot 1 \mathrm{M}$ citric acid effectively inhibits the formation of monetite phase that would otherwise be formed from the cement precursors at ambient laboratory conditions without any additives. ${ }^{30}$ Monetite formation was attributed in that study to the lack of stoichiometric water required for cement precursors to completely transform to brushite despite the crystal growth rate of brushite being several orders of magnitude higher than that of monetite. Bohner et al. conclude in their study on sodium citrate addition to brushite cements that low concentrations up to $0 \cdot 3 \mathrm{M}$ citric acid provide effective inhibition of monetite transformation due to small decrease in supersaturation. ${ }^{31}$ On the other hand, Hofmann's study on the effect of additives at much higher concentrations revealed that monetite content in the $\beta$-TCP-MCPM cement system was increased from 7 to $33 \%$ in expense of brushite, upon setting in aqueous solution containing 0.5 to $1.5 \mathrm{M}$ citric acid. ${ }^{32}$ Increase in monetite content was not attributed to insufficient water, as much lower powder/liquid ratio was used than the stoichiometric ratio of $4 \cdot 5$. The findings of our study conducted at dilute calcium phosphate concentrations show that citric acid concentrations $>0 \cdot 1 \mathrm{M}$ effectively inhibit brushite formation, while monetite content is not affected. However, it should be noted that the total citrate amount in excess water in our study is much higher than typical cement setting applications. Current study provides a borderline on the maximum effect of citric acid on phase composition of brushite cement. The ratio of monetite phase in concentrated CPC setting is seen to magnify due to much higher growth rates (E. Sahin and M. Ciftcioglu, unpublished work).

Citrate groups were explained to adsorb to the calcium phosphate surfaces through interaction with the calcium ions ${ }^{32}$ and the positively charged hydrated surfaces. ${ }^{7}$ Giocondi et al. revealed in their latest study that citrate molecules adsorb on brushite crystals rather as a surfactant than binding onto the dominant (010) surface sites due to the presence of a hydrate layer. ${ }^{7}$ The proposed mechanism of the inhibition of brushite growth in that study was adsorption on the hydrated surface and increase in the surface free energy, which in

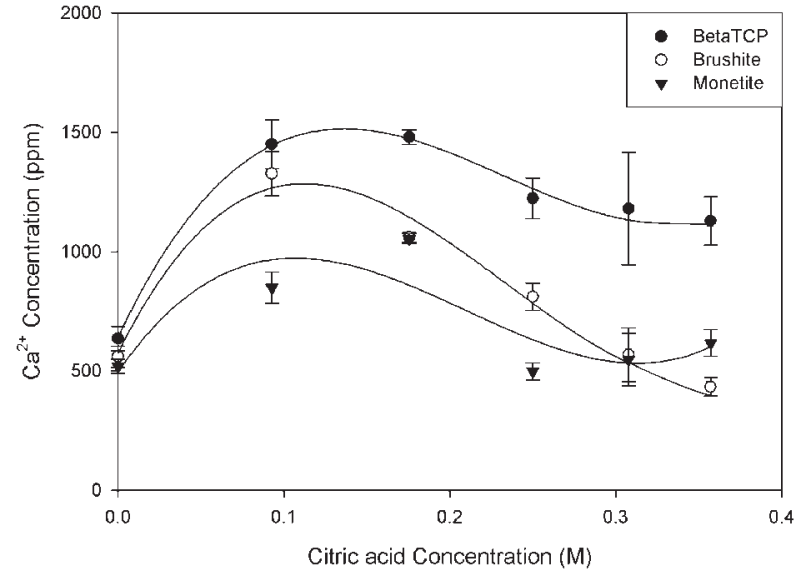

7 Free calcium concentrations of calcium phosphates in citrate solutions as determined by inductively coupled plasma atomic emission spectroscopy

turn resulted in a decrease in step density at low citrate concentrations. On the other hand at high citrate concentrations, citrate molecules bound onto the polar [101] calcium terminated steps. The SFM analysis by Flade et al. on adsorption of osteocalcin, another macromolecule with calcium affinity, on brushite surface shows that the calcium and phosphate ions in the brushite (010) plane are not able to interact with macromolecules. ${ }^{33}$ In comparison, monetite crystal structure excludes water molecules, and the surface is free from hydration, which exposes more adsorption sites for citrate molecules. ${ }^{9}$ The decrease in brushite/ monetite ratio observed with increasing citrate concentration can be explained by adsorption of citrate on brushite both as a calcium chelator and a surfactant, leading to a higher decrease in its solubility and growth compared to hydrate free monetite. This theory was tested using ICP atomic emission spectroscopy on the supernatant solutions of pure $\beta$-TCP, brushite and monetite in the presence of excess citrate molecules.

The $\mathrm{pH}$ of the citrate solutions was adjusted to $4 \cdot 2$, and equal amounts of calcium phosphates were mixed in the solutions containing various citric acid concentrations for $24 \mathrm{~h}$. The equilibrium $\mathrm{pH}$ of the calcium phosphate solutions in pure water reached the highest value for $\beta$-TCP due to its high solubility. $\beta$-TCP was also the phase with solubility most susceptible to $\mathrm{pH}$ variation. Initial low calcium concentration in all solutions was due to a difference in equilibrium $\mathrm{pH}$ of about $\Delta \mathrm{pH} 2 \cdot 0$ between pure water $(\mathrm{pH} \sim 7)$ and citric acid solution $(\mathrm{pH} \sim 5)$. Calcium concentrations of calcium phosphate solutions shown in Fig. 7 demonstrate the linear proportionality between $\beta$-TCP, brushite solubility and citric acid concentration $>0 \cdot 1 \mathrm{M}$ citric acid. The decreasing trend in calcium concentration was not observed for monetite as its calcium concentration remained in the same range at high citric acid concentrations. Brushite had the lowest solubility at the highest citric acid concentration contrary to the solubility isotherms observed in pure water. For comparison, solubility isotherms for the $\mathrm{CaOH}-\mathrm{H}_{2} \mathrm{O}-$ $\mathrm{H}_{3} \mathrm{PO}_{4}$ system dictate that in the acidic $\mathrm{pH}$ range, the solubility of the phases present in the system under study follows the order of ${ }^{34}$ : 


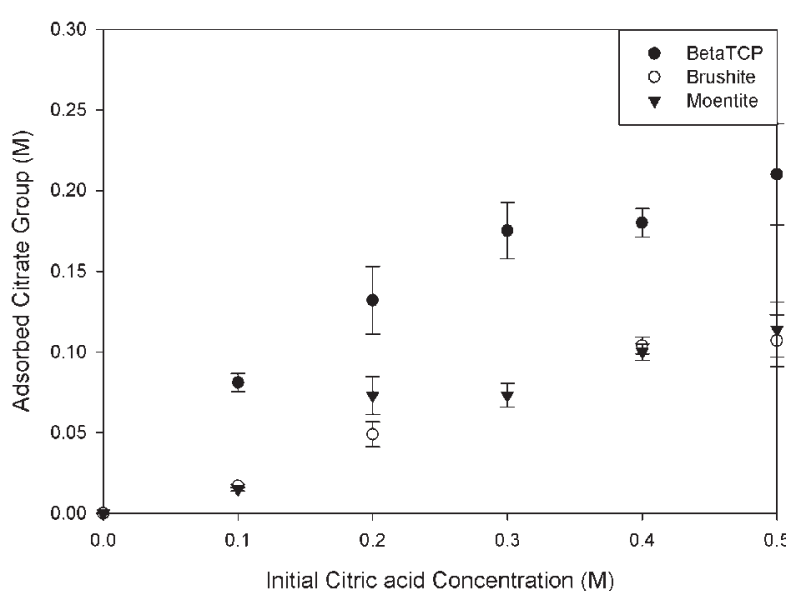

8 Net citrate adsorption on calcium phosphate species as function of initial citric acid concentration of aqueous calcium phosphate solutions

$\mathrm{MCPM}>>\beta$-TCP $>$ brushite $>$ monetite

Adsorption of citrate groups on calcium phosphates is proportional to the calcium/phosphate ratio at the surface of the calcium phosphate crystals, density of surface calcium ions and the magnitude of surface positive charge. $\beta$-TCP, which has a $\mathrm{Ca} / \mathrm{P}$ of $1 \cdot 5$, is known to host more citrate groups than brushite and monetite. ${ }^{8}$ Insensitivity of monetite solubility to citrate concentration as opposed to the linear dependence of brushite solubility to citrate concentration is attributed to the higher density of surface calcium ions on monetite crystals compared to the dominantly hydrated surface of brushite. Higher surface area of brushite powder used in the spectrometric analysis further supports this outcome. Specific surface areas of the powders determined by the Blaine method were $0.582 \mathrm{~m}^{2} \mathrm{~g}^{-1}$ for $\beta$-TCP, $0.462 \mathrm{~m}^{2} \mathrm{~g}^{-1}$ for brushite and $0.301 \mathrm{~m}^{2} \mathrm{~g}^{-1}$ for monetite. The highest solubility observed in the ICP analysis for $\beta$-TCP is partly due to the highest surface area of this powder.

It is known that changes occur at the surface of monetite when in contact with water at $37^{\circ} \mathrm{C}$, such that dissolution is hindered by slow topotactic growth of a nanometre thin calcium deficient apatite formation on the surface. ${ }^{10}$ This reduction in monetite dissolution is a function of time and is not expected to reduce the calcium concentration of samples prepared for ICP analysis due to the short period of analysis time. Despite their lower surface area, monetite crystals seem to accommodate more citrate ions and still release calcium ions, but brushite dissolution is confined to the step edges on the dominant (010) faces where calcium and phosphate ions are exposed to the solution. Surface exposed calcium ratio of brushite is expected to decrease linearly with increasing citrate concentrations, according to the trend given in Fig. 7, while that of monetite should be sufficiently high despite the slow formation of surface apatitic layer.

The mechanism of citrate adsorption on brushite and monetite was further investigated by measuring the concentration of citrate in the supernatants of aqueous calcium phosphate solutions via UV spectrophotometry. Similar adsorption capacities of equal masses of brushite

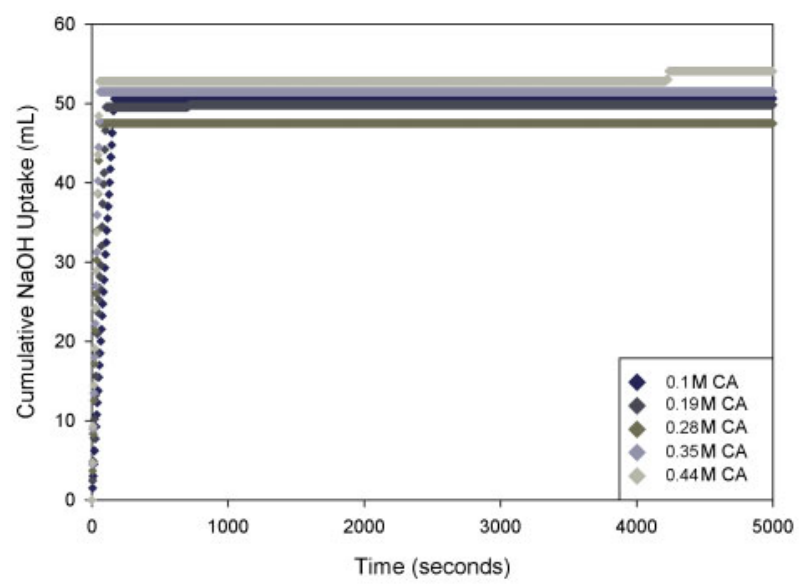

9 Cumulative base uptake curves for cement setting with various citric acid concentrations

and monetite phases seen in Fig. 8 do not reflect the higher surface calcium density of monetite because of the lower surface area of monetite powder used in the analysis. The lower citrate concentration in the $\beta$-TCP solution supernatant was mainly due to the higher $\mathrm{Ca} / \mathrm{P}$ ratio of that phase. The difference between the initially added citrate concentration and the measured concentration in the supernatant gives the net adsorption on the calcium phosphate phases. While small fluctuations in the net adsorption exists, brushite and monetite generally adsorb similar amounts of citrate despite the higher surface area of brushite used in the study. $\beta$-TCP crystals are able to accommodate most of the citrate added at the concentration range of $0 \cdot 1-0 \cdot 5 \mathrm{M}$, which explains the high $\beta$-TCP residue amount of cement precipitates set in solution containing $>0 \cdot 1 \mathrm{M}$ citric acid.

\section{pH stat runs}

The growth curves of all samples set in citric acid solution reach stability within the first few minutes of mixing in the solution. Figure 9 shows that even $0 \cdot 1 \mathrm{M}$ citric acid addition effectively inhibits any extended dissolution of $\beta$-TCP and the relaxation period is extremely compressed. High amount of base uptake was a result of neutralisation of the hydronium ions released from MCPM species in the absence of $\beta$-TCP dissolution. Almost all precipitation and setting is seen to occur in the first few minutes and abruptly ceases as the forming phase is saturated. Only samples set in $0.44 \mathrm{M}$ citric acid solution exhibited a portion of the secondary relaxation curve around $4000 \mathrm{~s}$, possibly due to high ionic strength inducing a suppression of the supersaturation of the thermodynamically stable monetite phase and kinetically favouring its formation. Increasing ionic strength is known to exponentially decrease both brushite and monetite supersaturations and the difference between them according to Pitzer's ion interaction model. ${ }^{35}$ Gradual decrease in the brushite/monetite ratio with increasing citric acid concentration seen in Fig. 10 indicates that thermodynamical stability of brushite approaches that of monetite, further decreasing its growth kinetics according to Oswald rule of stages. Phase evolution of brushite cement set under constant $\mathrm{pH}$ condition with increasing citric acid concentration shows that citrate adsorption inhibits both dissolution of $\beta$-TCP and growth of brushite. This adverse effect builds up with gradual 


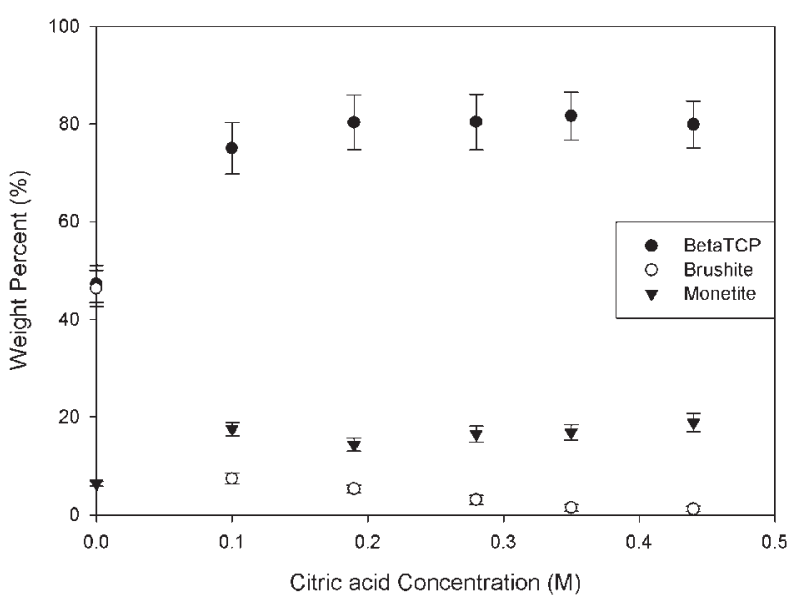

10 Phase composition of CPC set in presence of varying citric acid concentration

increase in citric acid concentration, while monetite formation extent is increased with the introduction of citric acid to aqueous setting solution and unaffected with further addition.

The weight percentages in Fig. 10 calculated by quantitative XRD do not show high conversion extent as the $\beta$-TCP residue amounts to $80 \%$. This is due to the overall suppression of $\beta$-TCP and brushite dissolution and growth due to excess citrate molecules. However, it should be considered that the volume of water used in $\mathrm{pH}$ stat and free drift runs was about 100 times larger than used in cement setting applications. Excessive dilution of the cement setting liquid lowered the acidity and supersaturations considerably with respect to the cement setting liquid added in clinical applications up to a powder/liquid ratio $\sim 4$. In the latter case, MCPM is also supersaturated and constantly acts to lower the $\mathrm{pH}$ by dissolution. The monetite conversion extent observed in these cement setting experiments is seen to reach $85 \%$ in our recent study since $\beta$-TCP solubility is exponentially proportional to acidity and monetite is known to be kinetically favoured to brushite at such acidic conditions (E. Sahin and M. Ciftcioglu, unpublished work). Moreover, impurity effectiveness of citrate molecules is much lower for cement setting with low liquid volume due to high calcium phosphate supersaturations and low citrate concentration. Therefore, another ionic species is needed to lower the calcium phosphate supersaturation and to enable more efficient citrate adsorption. $\mathrm{NaCl}$ is the most appropriate candidate to be used as the electrolyte for modification of the ionic strength of the cement system considering its singular monetite promoting effect. Preliminary data from the investigation of the synergistic effects of both citrate groups and $\mathrm{NaCl}$ on $\beta$-TCP-MCPM cement system at various concentrations reveal that $\mathrm{NaCl}$ can effectively balance the supersaturation to activate citrate groups in order to modify the phase composition of brushite cements in favour of monetite.

\section{Conclusions}

Citric acid addition to the brushite cement setting solution is seen to increase the monetite mass ratio. Citric acid altered the kinetics of brushite dissolution and growth and inhibited its formation, changing the order of formation in favour of monetite. Mechanism of the change in brushite cement setting kinetics by citrate adsorption is thought to be the relative stabilisation of brushite due to the relatively low calcium site density on the hydrated brushite crystal surface. Thus, supersaturation gap between brushite and monetite is expected to be narrower in the presence of excess citrate molecules due to their selective inhibition of brushite dissolution and growth. The results constitute the borderline for brushite cement setting at the extreme condition of excess citrate molecules. Monetite promoting effect of citric acid is expected to be enhanced in the case of clinical cement setting applications, where high calcium phosphate supersaturations enable higher growth rates. The synergistic interactions of citric acid with an ionic strength modifying electrolyte such as $\mathrm{NaCl}$ is worth further investigation considering the inefficiency of impurity adsorption at high growth rates.

\section{Acknowledgements}

The authors thank the facility and staff of Izmir Institute of Technology, Biotechnology and Bioengineering Research and Application Center and Materials Research Center for their contribution on the experimental work. We would also like to thank Çimentaş Cement Corporation for the surface area determination.

\section{References}

1. J. L. Ong and D. C. N. Chan: Critical Reviews in Biomedical Engineering, 2000, 28, (5/6), 667-707.

2. E. Rivera-Munoz, J. R. Diaz, J. Rogelio Rodriguez, W. Brostow and V. M. Castano: J. Mater. Sci.: Mater. Med., 2001, 12, (4), 305-311.

3. M. Bohner: Mater. Today, 2010, 13, (1-2), 24-30.

4. M. Bohner: Injury, 2000, 31, (Suppl 4), 37-47.

5. A. Sáenz, E. Rivera, W. Brostow and V. M. Castano: J. Mater. Educ., 1999, 21, (5/6), 267-276.

6. K. Flade, C. Lau, M. Mertig and W. Pompe: Chem. Mater., 2001, 13, (10), 3596-3602.

7. J. L. Giocondi, B. S. El-Dasher, G. H. Nancollas and C. A. Orme: Philos. Trans. R. Soc. Lond. A, 2010, 368A, (1917), 1937-1961.

8. M. H. Alkhraisat, F. T. Marino, J. R. Retama, L. B. Jerez and E. Lopez-Cabarcos: J. Biomed. Mater. Res. A, 2008, 84A, (3), 710717.

9. L. Tortet, J. R. Gavarri, G. Nihoul and A. J. Dianoux: J. Solid State Chem., 1997, 132, (1), 6-16.

10. A. Lebugle, B. Sallek and A. T. Tai: J. Mater. Chem., 1999, 9, (10), 2511-2515.

11. M. Catti, G. Ferraris and A. Filhol: Acta Crystallogr. B, 1977, 33B, (4), 1223-1229.

12. P. F. Schofield, K. S. Knight, J. A. M. van der Houwen and E. Valsami-Jones: Phys. Chem. Miner., 2004, 31, (9), 606-624.

13. D. Barthelmy: 'Mineralogy database', 2012. http://www.webmineral. com/data/Monetite.shtml (accessed 5 September 2012).

14. L. G. Galea, M. Bohner, J. Lemaitre, T. Kohler and R. Muller: Biomaterials, 2008, 29, (24-25), 3400-3407.

15. J. Aberg, H. Brisby, H. B. Henriksson, A. Lindahl, P. Thomsen and H. Engqvist: J. Biomed. Mater. Res. B, 2010, 93B, (2), 436-441.

16. M. Bohner, H. P. Merkle and J. Lemaitre: J. Mater. Sci.: Mater. Med., 2000, 11, (3), 155-162.

17. L. M. Grover, U. Gbureck, A. M. Young, A. J. Wright and J. E. Barralet: J. Mater. Chem., 2005, 15, (46), 4955-4962.

18. F. Tamimi, Z. Sheikh and J. Barralet: Acta Biomater., 2012, 8, (2), 474487.

19. M. Bohner, H. P. Merkle, P. V. Landuyt, G. Trophardy and J. Lemaitre: J. Mater. Sci., Mater. Med., 1999, 11, (2), 111-116.

20. M. H. Alkhraisat, F. T. Marino, C. R. Rodriguez, L. B. Jerez and E. L. Cabarcos: Acta Biomater., 2008, 4, (3), 664-670.

21. C. A. Orme and J. L. Giocondi: 'Model systems for formation and dissolution of calcium phosphate minerals', in 'Handbook of biomineralization: biomimetic and bioinspired chemistry'; 2007, New York, Wiley. 151-155. 
22. R. K. Tang, M. Darragh, C. A. Orme, X. Y. Guan, J. R. Hoyer and G. H. Nancollas: Angew. Chem. Int. Ed., 2005, 44, (24), 36983702 .

23. R. Rosmaninho and L. F. Melo: J. Food Eng., 2006, 73, (4), 379387

24. A. Mersmann: 'Crystallization technology handbook'; 2001, New York, Marcel-Dekker, Inc.

25. B. D. Cullity: 'Elements of X-ray diffraction'; 1956, Reading, MA, Addison-Wesley.

26. R. L. Snyder: 'Introduction to X-ray powder diffractometry'; 1996 , New York, Wiley-Interscience.

27. L. Wang and G. H. Nancollas: Chem. Rev., 2008, 108, (11), 46284669

28. M. Bohner and U. Gbureck: J. Biomed. Mater. Res. B, 2008, 84B, (2), 375-385.
29. M. Bohner, P. van Landuyt, H. P. Merkle and J. Lemaitre: J. Mater. Sci.: Mater. Med., 1997, 8, (11), 675-681.

30. U. Gbureck, S. Dembski, R. Thull and J. E. Barralet: Biomaterials, 2005, 26, (17), 3691-3697.

31. M. M. Bohner, P. van Landuyt, P. Trophardy and G. Lemaitre: J. Mater. Sci.: Mater. Med., 2000, 11, 111-116.

32. M. P. Hofmann, A. M. Young, U. Gbureck, S. N. Nazhat and J. E. Barralet: J. Mater. Chem., 2006, 16, (31), 3199-3206.

33. K. Flade, C. Lau, M. Mertig and W. Pompe: Chem. Mater., 2001, 13, (10), 3596-3602.

34. E. Fernandez, F. J. Gil, M. P. Ginebra, F. C. Driessens, J. A. Planell and S. M. Best: J. Mater. Sci.: Mater. Med., 1999, 10, (3), 169-176.

35. E. Sahin and M. Ciftcioglu: J. Mater. Chem. B, 2013, 1, (23), 29432950. 